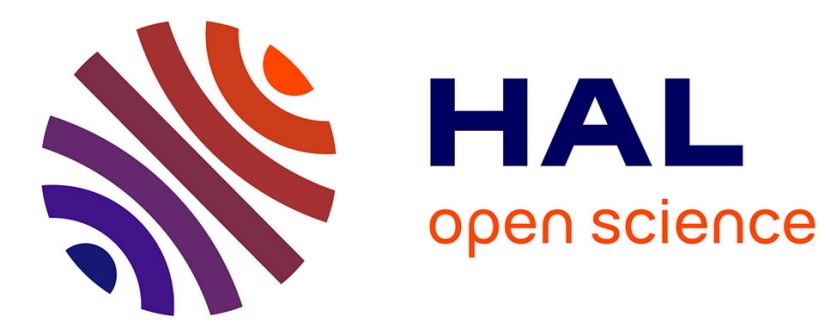

\title{
Randomness and the ergodic decomposition
}

\author{
Mathieu Hoyrup
}

\section{To cite this version:}

Mathieu Hoyrup. Randomness and the ergodic decomposition. Computability in Europe, Jun 2011,

Sofia, Bulgaria. pp.122-131. inria-00586736

\section{HAL Id: inria-00586736 \\ https://hal.inria.fr/inria-00586736}

Submitted on 18 Apr 2011

HAL is a multi-disciplinary open access archive for the deposit and dissemination of scientific research documents, whether they are published or not. The documents may come from teaching and research institutions in France or abroad, or from public or private research centers.
L'archive ouverte pluridisciplinaire HAL, est destinée au dépôt et à la diffusion de documents scientifiques de niveau recherche, publiés ou non, émanant des établissements d'enseignement et de recherche français ou étrangers, des laboratoires publics ou privés. 


\title{
Randomness and the ergodic decomposition
}

\author{
Mathieu Hoyrup ${ }^{1}$ \\ LORIA, INRIA Nancy-Grand Est, 615 rue du jardin botanique, BP 239, 54506 \\ Vandœuvre-lès-Nancy, France, mathieu.hoyrup@loria.fr
}

\begin{abstract}
The interaction between algorithmic randomness and ergodic theory is a rich field of investigation. In this paper we study the particular case of the ergodic decomposition. We give several positive partial answers, leaving the general problem open. We shortly illustrate how the effectivity of the ergodic decomposition allows one to easily extend results from the ergodic case to the non-ergodic one (namely Poincaré recurrence theorem). We also show that in some cases the ergodic measures can be computed from the typical realizations of the process.
\end{abstract}

Keywords: computable analysis, Martin-Löf randomness, ergodic decomposition, Birkhoff's ergodic theorem.

\section{Introduction}

The goal of the paper is to study the interaction between the theory of algorithmic randomness, started by Martin-Löf [9], and ergodic theory (i.e. restricting to shift-invariant measures). The first results in this direction were obtained by V'yugin [12], who proved that Birkhoff's ergodic theorem and a weak form of Shannon-McMillan-Breiman theorem hold for each Martin-Löf random sequence. Recently several improvements of the first result have been achieved [10,6,1].

A classical result from ergodic theory, called the ergodic decomposition, states that given a stationary process, almost every realization is actually a typical realization of an ergodic process. The full process can be decomposed as the combination of a collection of ergodic processes. It is natural to ask the question whether every Martin-Löf random sequence (with respect to the stationary measure) statistically induces an ergodic measure, and if the sequence is Martin-Löf random with respect to it.

We give three orthogonal cases in which we can give a positive answer: (i) when the decomposition of the measure is computable, (ii) when the decomposition of the measure is supported on an effective compact class of ergodic measures, (iii) when the decomposition of the measure is finite. Observe that the three cases are mutually incomparable. We leave the general problem open.

As a side result, we give sufficient conditions to infer the statistics of the system from the observation; formally we give a sufficient condition on an ergodic measure to be computable relative to its random elements.

In Section 2 we give the necessary background on computability and randomness. In Section 3 we develop results about randomness and combinations 
of measures that will be applied in the sequel, but are of independent interest (outside ergodic theory). We start Section 4 with a reminder on the ergodic decomposition and then we present our results relating it to randomness.

\section{Preliminaries}

We assume familiarity with algorithmic randomness and computability theory.

All the results stated in this paper hold on effectively compact computable metric spaces $X$ and for computable maps $T: X \rightarrow X$ (as defined in computable analysis, see [13]), but for the sake of simplicity we formulate them only on the Cantor space $X=\{0,1\}^{\mathbb{N}}$ and for the shift transformation $T: X \rightarrow X$ defined by $T\left(x_{0} x_{1} x_{2} \ldots\right)=x_{1} x_{2} x_{3} \ldots$. The Cantor space is endowed with the product topology, generated by the cylinders $[w], w \in\{0,1\}^{*}$. Implicitly, measures are probability measures defined on the Borel $\sigma$-algebra, and ergodic measures are stationary (i.e., shift-invariant) ergodic Borel probability measures. The set $\mathcal{P}(X)$ of probability measures over $X$ is endowed with the weak* topology, given by the notion of weak convergence: measures $P_{n}$ converge to $P$ if for every $w \in\{0,1\}^{*}$, $P_{n}[w] \rightarrow P[w]$.

A name for a real number $r$ is an infinite binary sequence encoding, in some canonical effective way, a sequence of rational numbers $q_{n}$ such that $\left|q_{n}-r\right|<2^{-n}$ for all $n$. A name for a probability measure $P$ is the interleaving, in some canonical effective way, of names for the numbers $P[w], w \in\{0,1\}^{*}$. A computable probability measure is a measure admitting a computable name: in other words, the numbers $P[w]$ are uniformly computable.

Let $X, Y$ be any spaces among $\{0,1\}^{\mathbb{N}}, \mathbb{R}$ and $\mathcal{P}(X)$. A function $f: X \rightarrow Y$ is computable if there is an oracle machine that, given a name of $x \in X$ as an oracle, outputs a name of $f(x)$ (the computation never halts). Computable functions are continuous. $f$ is computable on a set $A \subseteq X$ if the same holds for all $x \in A$ (nothing is required to the machine when $x \notin A$ ). An object $y$ is computable relative to an object $x$ if the function $x \mapsto y$ is computable on $\{x\}$, i.e. if there is an oracle machine that on any name of $x$ as oracle, produces a name of $y$.

An open subset $U$ of the Cantor space is effective if there is a (partial) computable function $\varphi: \mathbb{N} \rightarrow\{0,1\}^{*}$ such that $U=\bigcup_{n \in \mathbb{N}}[\varphi(n)]$. An effective compact set is the complement of an effective open set. Let $K \subseteq X$ be an effective compact set and $f: K \rightarrow Y$ a function computable on $K$.

Fact 1 (Folklore) $f(K)$ is an effective compact set.

Fact 2 (Folklore) If $f$ is moreover one-to-one then $f^{-1}: f(K) \rightarrow K$ is computable on $f(K)$.

The product of two computable metric spaces has a natural structure of computable metric space.

Fact 3 (Folklore) If $K \subseteq X$ is an effective compact set and $f: K \times Y \rightarrow$ $\overline{\mathbb{R}}$ is lower semi-computable, then the function $g: Y \rightarrow \overline{\mathbb{R}}$ defined by $g(y)=$ $\inf _{x \in K} f(x, y)$ is lower semi-computable. 
If $f, g$ are real-valued functions, $f \stackrel{*}{<} g$ means that there exists $c \geq 0$ such that $f \leq c g . f \stackrel{*}{=} g$ means that $f \stackrel{*}{<} g$ and $g \stackrel{*}{<} f$.

\section{$2.1 \quad$ Effective randomness}

Martin-Löf [9] was the first one to define a sound individual notion of random infinite binary sequence. He developed his theory for any computable probability measure on the Cantor space. This theory was then extended to non-computable measures by Levin [8], and later by [3,7] on general spaces ([5] was an extension to topological spaces, but for computable measures).

We will use the most general theory: we will be interested in randomness on the Cantor space and on the space of Borel probability measures over the Cantor space, for arbitrary (i.e. not necessarily computable) probability measures. In particular, we will use the notion of uniform test of randomness, introduced by Levin [8] and further developed in $[3,4,7]$.

On a computable metric space $X$ endowed with a probability measure $P$, there is a set $\mathcal{R}_{P}$ of $P$-random elements satisfying $P\left(\mathcal{R}_{P}\right)=1$, together with a canonical decomposition (coming from the universal $P$-test) $\mathcal{R}_{P}=\bigcup_{n} \mathcal{R}_{P}^{n}$ where $\mathcal{R}_{P}^{n}$ are uniformly effective compact sets relative to $P, \mathcal{R}_{P}^{n} \subseteq \mathcal{R}_{P}^{n+1}$ and $P\left(\mathcal{R}_{P}^{n}\right)>1-2^{-n}$. The sets $X \backslash \mathcal{R}_{P}^{n}$ constitute a universal Martin-Löf test. A $P$-test is a function $t: X \rightarrow[0,+\infty]$ which is lower semi-computable relative to $P$, such that $\int t \mathrm{~d} P \leq 1$.

A function $f: X \rightarrow Y$ is P-layerwise computable if there is an oracle machine that, given $n$ as input and a name of $x \in \mathcal{R}_{P}^{n}$ as an oracle, outputs a name of $f(x)$ (the computation never halts). Nothing is required to the machine when $x$ is not $P$-random. When $f$ is $P$-layerwise computable, for every $P$-random $x, f(x)$ is computable relative to $x$ in a way that is not fully uniform, but uniform on each set $\mathcal{R}_{P}^{n}$.

Such a machine can be thought of as a probabilistic algorithm, but here the randomness is not part of the algorithm but of the input. Formally, it is the same notion, but usually, "succeeding with high probability" means that if we run the program on a given input several times, independently, it will succeed most of the times; here, the algorithm is deterministic and it will succeed on most inputs.

Lemma 1. Let $P$ be a computable measure, $f: X \rightarrow Y$ a P-layerwise computable function and $Q=f_{*} P$ the push-forward of $P$ under $f$.

1. $Q$ is computable and $f: \mathcal{R}_{P} \rightarrow \mathcal{R}_{Q}$ is onto.

2. If $f: X \rightarrow Y$ is moreover one-to-one then $f: \mathcal{R}_{P} \rightarrow \mathcal{R}_{Q}$ is one-to-one and $f^{-1}$ is Q-layerwise computable.

Proof. We only prove that $f^{-1}$ is $Q$-layerwise computable, the other statements are proved in [6]. There is $c \in \mathbb{N}$ such that $\mathcal{R}_{Q}^{n} \subseteq f\left(\mathcal{R}_{P}^{n+c}\right)$ for all $n$. Let $n \in \mathbb{N}$. $f: \mathcal{R}_{P}^{n+c} \rightarrow Y$ is one-to-one and computable so by Fact $2, f^{-1}: f\left(\mathcal{R}_{P}^{n+c}\right) \rightarrow X$ is computable. As $\mathcal{R}_{Q}^{n} \subseteq f\left(\mathcal{R}_{P}^{n+c}\right), f^{-1}: \mathcal{R}_{Q}^{n} \rightarrow X$ is computable. This is uniform in $n$. 


\section{Randomness and continuous combination of measures}

The material developed here will be used to investigate the algorithmic content of the ergodic decomposition.

Given a countable class of probability measures $P_{i}$ and real numbers $\alpha_{i} \in$ $[0,1]$ such that $\sum_{i} \alpha_{i}=1$, the convex combination $P=\sum_{i} \alpha_{i} P_{i}$ is again a probability measure. This can be generalized to continuous classes of measures, as we briefly recall now.

Let $m$ be a probability measure over $\mathcal{P}(X)$. The set function $P$ defined by $P(A)=\int Q(A) \mathrm{d} m(Q)$ for measurable sets $A$ is a probability measure over $X$, called the barycenter of $m$. It satisfies $\int f \mathrm{~d} P=\int\left(\int f \mathrm{~d} Q\right) \mathrm{d} m(Q)$ for $f \in$ $L^{1}(X, P)$. When $m$ is computable, so is $P$. We can think of $P$ as the measure describing the following process: first pick some measure $Q$ at random according to $m$; then run the process with distribution $Q$.

Probabilistically, picking a sequence according to $P$ or decomposing into these two steps are equivalent. We are interested in whether the algorithmic theory of randomness fits well with this intuition: are the $P$-random sequences the same as the sequences that are $Q$-random for some $m$-random $Q$ ?

Remark 1. Let $f: X \rightarrow[0,+\infty]$ be a lower semi-computable function. Let $F: \mathcal{P}(X) \rightarrow[0,+\infty]$ be defined by $F(Q)=\int f \mathrm{~d} Q . F$ is lower semi-computable and $\int F \mathrm{~d} m=\int f \mathrm{~d} P$. As a result, $F$ is a $m$-test if and only if $f$ is a $P$-test.

Theorem 1. Let $m \in \mathcal{P}(\mathcal{P}(X))$ be computable, and $P$ be the barycenter of $m$. For $x \in X$, the following are equivalent:

1. $x$ is P-random,

2. $x$ is $Q$-random for some $m$-random $Q$.

In other words,

$$
\mathcal{R}_{P}=\bigcup_{Q \in \mathcal{R}_{m}} \mathcal{R}_{Q} .
$$

Proof.

Let $f(x)=\inf _{Q} t_{m}(Q) \cdot t_{Q}(x) . f$ is lower semi-computable by Fact $3(\mathcal{P}(X)$ is effectively compact). As $\int f \mathrm{~d} P=\int\left(\int f \mathrm{~d} Q\right) \mathrm{d} m(Q) \leq \int t_{m}(Q)\left(\int t_{Q} \mathrm{~d} Q\right) \mathrm{d} m(Q) \leq$ $1, f$ is a $P$-test, so if $x$ is $P$-random then it is $Q$-random for some $m$-random measure $Q$.

Conversely, let $T_{P}(Q)=\int t_{P} \mathrm{~d} Q$ where $t_{P}$ is a universal $P$-test. By Remark $1, T_{P}$ is an $m$-test so if $Q$ is $m$-random then $T_{P}(Q)<\infty$, so $t_{P}$ is a (multiple of a) $Q$-test. As a result, $\mathcal{R}_{Q} \subseteq \mathcal{R}_{P}$.

\section{Randomness and ergodic decomposition}

\subsection{Background from ergodic theory}

A sequence $x \in\{0,1\}^{\mathbb{N}}$ is generic if for each $w \in\{0,1\}^{*}$, the frequency of occurrences of $w$ in $x$ converges. If $x$ is generic, we denote by $Q_{x}$ the set function 
which maps each cylinder $[w]$ to the limit frequency of occurrences of $w$ in $x$. $Q_{x}$ extends to a probability measure over the Cantor space, which we also denote by $Q_{x}$. If $x$ is generic then $Q_{x}$ is stationary, i.e. $Q_{x}(A)=Q_{x}\left(T^{-1}(A)\right)$ for every measurable set $A$. Birkhoff's ergodic theorem states that given a stationary measure $P, P$-almost every $x$ is generic. A stationary measure $P$ is ergodic if the only invariant sets have measure 0 or 1 . Formally, if $T^{-1}(A)=A$ then $P(A)=0$ or 1 , for every measurable set $A$. If $P$ is stationary ergodic then $Q_{x}=P$ for $P$-almost every $x$.

The ergodic decomposition theorem states that given a stationary probability measure $P$, the measure $Q_{x}$ is ergodic for $P$-almost every $x$. There are mainly two proofs of this fact. One of them uses Choquet theorem from convex analysis (see [11]): the set of stationary probability measures is a convex compact set whose extreme points are exactly the ergodic measures. Then any point in that set, i.e. any invariant measure, can be expressed as a barycenter over the ergodic measures. More precisely, for any invariant measure $P$ there is a unique probability measure $m_{P}$ over $\mathcal{P}(X)$ which gives full weight to the ergodic measures, and such that $P(A)=\int Q(A) \mathrm{d} m_{P}(Q)$ for every Borel set $A$. We will call $m_{P}$ the Choquet measure associated to $P$.

\subsection{Randomness and ergodic theorems}

An algorithmic version of Birkhoff's ergodic theorem was eventually proved by V'yugin [12]: given a shift-invariant probability measure $P$, every $P$-random sequence is generic, and if $P$ is moreover ergodic then $Q_{x}=P$ for every $P$ random sequence $x$ (it was proved for computable measures, but it still works for non-computable measures). The proof was not immediate to obtain from the classical proof of Birkhoff's theorem, which is in a sense not constructive. In this paper we are interested in an algorithmic version of the ergodic decomposition theorem, which again cannot be proved directly.

More precisely, given a stationary measure $P$, we are interested in the following questions:

- if $x$ is $P$-random, is $Q_{x}$ ergodic?

- if $x$ is $P$-random, is $x$ also $Q_{x}$-random?

- if $x$ is $P$-random, is $Q_{x}$ an $m_{P}$-random measure?

- does any converse implication hold?

We give positive partial answers to these questions, leaving the general problem open. We will use the following lemmas (the first one was proved in [12]).

Lemma 2. Let $P$ be an ergodic stationary probability measure. For every $x \in$ $\mathcal{R}_{P}, Q_{x}=P$.

Lemma 3. Let $P$ be a stationary probability measure and $m_{P}$ the associated Choquet measure. Every $m_{P}$-random measure is ergodic and stationary.

Proof. It is known that the set of ergodic stationary measure is a $G_{\delta}$-set. It is moreover effective, i.e. it is an intersection of effective open sets. As it has $m_{P}$-measure one, it contains $\mathcal{R}_{m_{P}}$. 


\subsection{First answer: effective decomposition}

A stationary probability measure $P$ is always computable relative to its associated Choquet measure $m_{P}$. The converse does not always hold (see Section 4.4 for a counter-example).

Definition 1. A computable stationary probability measure $P$ is effectively decomposable if its Choquet measure is computable.

As an application of Theorem 1, we directly get a result when $P$ is effectively decomposable (i.e. when $m:=m_{P}$ is computable).

Corollary 1. Let $P$ be a computable stationary probability measure that is effectively decomposable. For $x \in X$, the following are equivalent:

1. $x$ is P-random,

2. $x$ is $Q$-random for some $m$-random $Q$.

In other words, the following are equivalent:

1. $x$ is P-random,

2. $x$ is generic, $Q_{x}$-random and $Q_{x}$ is m-random.

We also have the following characterization.

Theorem 2. Let $P$ be a computable stationary probability measure. The following are equivalent.

1. $P$ is effectively decomposable,

2. the function $X \rightarrow \mathcal{P}(X), x \mapsto Q_{x}$ is P-layerwise computable.

Proof. $1 \Rightarrow 2$. In any probability space $(Y, \mu)$ with random elements $\mathcal{R}_{\mu}=$ $\bigcup_{n} \mathcal{R}_{\mu}^{n}$, we define $d_{\mu}(y)=\min \left\{n: y \in \mathcal{R}_{\mu}^{n}\right\}\left(d_{\mu}(y)=+\infty\right.$ if $y$ is not $\mu$-random). $d: \mathcal{P}(Y) \times Y \rightarrow[0,+\infty]$ which maps $(\mu, y)$ to $d_{\mu}(y)$ is lower semi-computable.

Let $C_{n}=\left\{(Q, x): d_{m}(Q) \leq n\right.$ and $\left.d_{Q}(x) \leq n\right\}$. The second projection $\pi_{2}$ : $\bigcup_{n} C_{n} \rightarrow X$ is one-to-one. Indeed, if $\left(Q_{i}, x_{i}\right) \in \bigcup_{n} C_{n}, i=1,2$ and $\pi_{2}\left(Q_{1}, x_{1}\right)=$ $\pi_{2}\left(Q_{2}, x_{2}\right)$ then (i) $x_{1}=x_{2}$, (ii) $Q_{1}, Q_{2}$ are $m$-random hence ergodic, (iii) $x_{i}$ is $Q_{i}$-random so $Q_{x_{i}}=Q_{i}$; as a result, $Q_{1}=Q_{x_{1}}=Q_{x_{2}}=Q_{2}$. $C_{n}$ is effectively compact so $\pi_{2}^{-1}$ is computable on each $\pi_{2}\left(C_{n}\right)$ (uniformly in $n$ ) by Fact 2.

We know from the proof of Theorem 1 that there exists a constant $c$ such that for all $n$ and all $x \in \mathcal{R}_{P}^{n},\left(Q_{x}, x\right) \in C_{n+c}$, hence $\mathcal{R}_{P}^{n} \subseteq \pi_{2}\left(C_{n+c}\right)$. It implies that $\pi_{2}^{-1}$ is computable on each $\mathcal{R}_{P}^{n}$, uniformly in $n$, i.e. $\pi_{2}^{-1}$ is $P$-layerwise computable. Finally, $\pi_{1} \circ \pi_{2}^{-1}$, which maps $x \in \mathcal{R}_{P}$ to $Q_{x}$ is $P$-layerwise computable.

$2 \Rightarrow 1$. Conversely, if $\psi: x \mapsto Q_{x}$ is $P$-layerwise computable, then $m=\psi_{*} P$ is the push-forward of $P$ under $\psi$, so it is computable by Lemma 1, item 1 .

Remark 2. For $f \in L^{1}(X, P)$, we denote by $f^{*}$ the limit of the Birkhoff averages of $f$. One can also prove that if $P$ is computable then $P$ is effectively decomposable if and only if the function

$$
\begin{aligned}
L^{1}(X, P) & \rightarrow L^{1}(X, P) \\
f & \mapsto f^{*}
\end{aligned}
$$

is computable. 
The effectivity of the ergodic decomposition enables one to extend results from ergodic systems to non-ergodic ones. Let us illustrate it. It was proved in [1] that when $P$ is an ergodic measure, every $P$-random sequence eventually visits every effective compact set of positive measure under shift iterations. When the decomposition is effective, this theorem can be generalized to non-ergodic measures, giving a version of Poincaré recurrence theorem for random sequences.

Corollary 2. Let $P$ be a stationary measure that is effectively decomposable. Let $F$ be an effective compact set such that $P(F)>0$. Every $P$-random $x \in F$ falls infinitely often in $F$ under shift iterations.

Proof. $x$ is $Q_{x}$-random and $Q_{x}$ is ergodic. As all random sequences belong to effective open sets of measure one and $x \in F, Q_{x}(F)>0$. Hence we can apply the result in [1] to the ergodic measure $Q_{x}$ (strictly speaking their result was proved for computable ergodic measures, but it can be relativized without difficulty).

The result actually holds as soon as for every $P$-random $x, Q_{x}$ is ergodic and $x$ is $Q_{x}$-random.

\subsection{V'yugin's example}

In [12], V'yugin constructed a computable stationary measure for which the convergence of Birkhoff's average is not effective. We give a simpler construction and show that this measure is not effectively decomposable.

Let $M_{i}$ be some effective enumeration of the Turing machines. For each $i$, let $p_{i}=2^{-t_{i}}$ if $M_{i}$ halts in time $t_{i}, p_{i}=0$ if $t_{i}$ does not halt. The real numbers $p_{i}$ are computable uniformly in $i$ (while they are not uniformly computable as rational numbers). Let $P_{i}$ be the Markovian stationary measure defined by $P_{i}[0]=P_{i}[1]=\frac{1}{2}$ and $\frac{P_{i}[w 01]}{P_{i}[w 0]}=\frac{P_{i}[w 10]}{P_{i}[w 1]}=p_{i}$ for all $w \in\{0,1\}^{*}$ (the probability of changing between states 0 and 1 is $\left.p_{i}\right)$. Let $P=\sum_{i} 2^{-i} P_{i}$. $P$ is computable. We now show that $x \mapsto Q_{x}$ is not $P$-layerwise computable (which will imply that $P$ is not effectively decomposable by Theorem 2). Let $f=\chi_{[1]}$. Let $\alpha=$ $\sum_{i: M_{i} \text { halts }} 2^{-i} \cdot f^{*}(x)=0$ for $x=0^{\mathbb{N}}, f^{*}(x)=1$ for $x=1^{\mathbb{N}}$ and $f^{*}(x)=\frac{1}{2}$ for $P$ almost all $x \notin\left\{0^{\mathbb{N}}, 1^{\mathbb{N}}\right\}$. By definition of $Q_{x}, f^{*}(x)=Q_{x}[1]$ for every $P$-random $x$. If $f^{*}$ were $P$-layerwise computable, then $P\left(f^{*-1}[0,1 / 4)\right)=(1-\alpha) / 2$ would be lower semi-computable by basic properties of layerwise computable functions (see $[6]$ ).

While $P$ is not effectively decomposable, we can still get a result about random elements.

Proposition 1. For every P-random $x, Q_{x}$ is ergodic and $x$ is $Q_{x}$-random.

Proof. The decomposition $P=\sum_{i} 2^{-i} P_{i}$ is partial in the sense that some $P_{i}$ are not ergodic (when $M_{i}$ does not halt). However we can apply Theorem 1 to this decomposition: $P$ is the barycenter of the computable measure $m^{\prime}=\sum_{i} 2^{-i} \delta_{P_{i}}$, so every $P$-random $x$ is random for some $P_{i}$. (i) If $M_{i}$ halts, then $P_{i}$ is ergodic. (ii) If $M_{i}$ does not halt then $P_{i}=\frac{1}{2}\left(\delta_{0}+\delta_{1}\right)$ (where $\delta_{0}$ is the measure concentrated 
on $0^{\mathbb{N}}, \delta_{1}$ on $\left.1^{\mathbb{N}}\right)$. In turn, $P_{i}$, which is non-ergodic is effectively decomposable. Hence as $x$ is $P_{i}$-random, $Q_{x}=\delta_{0}$ or $\delta_{1}$ and $x$ is $Q_{x}$-random.

As a result, Corollary 2 also holds for the measure $P$.

\subsection{A particular case: effective compact classes of ergodic measures}

Proposition 2. Let $P$ be a computable stationary probability measure. If $m_{P}$ is supported on an effective compact class of ergodic measures, then $P$ is effectively decomposable.

Proof. Let $\mathscr{C}$ be an effective compact class of stationary ergodic probability measures. Let $\mathcal{P}(\mathscr{C})$ be the set of probability measures $m$ over $\mathcal{P}(X)$ such that $m(\mathscr{C})=1 . \mathcal{P}(\mathscr{C})$ is an effective compact subset of $\mathcal{P}(X)$ : indeed, it is the preimage of $[1,+\infty]$ under the upper semi-computable function $m \mapsto m(\mathscr{C})$. If $m \in \mathcal{P}(\mathscr{C})$, the barycenter $P$ of $m$ is defined by $P(A)=\int Q(A) \mathrm{d} m(Q)$ for every measurable set $A$. The function $\psi$ which maps $m$ to $P$ is computable. Let $\mathcal{J}_{\mathscr{C}}$ be the class of invariant measures that are barycenters of $\mathscr{C}$, i.e. the image of $\mathcal{P}(\mathscr{C})$ under $\psi: \mathcal{J}_{\mathscr{C}}$ is an effective compact class too. By existence and uniqueness of the ergodic decomposition, $\psi: \mathcal{P}(\mathscr{C}) \rightarrow \mathcal{J}_{\mathscr{C}}$ is onto and one-to-one; as it is computable and $\mathcal{P}(\mathscr{C})$ is an effective compact set, its inverse is also computable by Fact 2 .

The above proposition implies the computability of De Finetti measures on the Cantor space (see [2]).

Example 1. Let $m$ be a computable probability measure over the real interval $[0,1]$. Pick a real number $p$ at random according to $m$, and then generate an infinite sequence of 0,1 tossing a coin with probability of heads $p$. As an application of the preceding proposition, we get that the function which maps a random sequence generated by the process to the number $p$ that was picked is $P$-layerwise computable: it can be computed from the observed outcomes with high probability.

We also learn that the algorithmic theory of randomness fits well with this example: obviously, we expect a random sequence for the whole process to be random for some Bernoulli measure $B_{p}$, which is not immediate.

In Section 2.1, we define $P$-layerwise computable function when $P$ is a computable probability measure. This can be extended straightforwardly to any effective compact class of measures $\mathscr{C}$. The universal $\mathscr{C}$-test induces a decomposition $\bigcup_{n} \mathcal{R}_{n}^{\mathscr{C}}$ of the sequences that are random for some measure in $\mathscr{C}$. A function $f: X \rightarrow Y$ is $\mathscr{C}$-layerwise computable if it is computable on each $\mathcal{R}_{n}^{\mathscr{C}}$, uniformly in $n$. It means that one can compute $f(x)$ if $x$ is random for some measure $P \in \mathscr{C}$, with probability of error bounded by $2^{-n}$, whatever $P$ is (as long as it is in $\mathscr{C}$ ), and for any $n$.

From Proposition 2 and Corollary 1 we know that for every $P \in \mathcal{J}_{\mathscr{C}}$ and every $x \in \mathcal{R}_{P}, Q_{x}$ is $m$-random, hence ergodic and $x$ is $Q_{x}$-random. We also 
prove a quantitative version of this fact. We recall that if $\mathcal{A}$ is an effective compact class of measures, $t_{\mathcal{A}}:=\inf _{P \in \mathcal{A}} t_{P}$ is a universal $\mathcal{A}$-test, i.e. (i) it is lower semi-computable, (ii) $\int t_{\mathcal{A}} \mathrm{d} P \leq 1$ for every $P \in \mathcal{A}$ and (iii) $t_{\mathcal{A}}$ multiplicatively dominates every function satisfying (i) and (ii) (see [4] for more details about such class tests). We will consider the class tests $t_{\mathscr{C}}$ and $t_{\mathfrak{J}_{\mathscr{C}}}$.

Theorem 3. Let $\mathscr{C}$ be an effective compact class of stationary ergodic probability measures. One has:

1. $t_{\mathscr{C}}(x) \stackrel{*}{=} t_{\mathfrak{J}_{\mathscr{C}}}(x)$

2. The function $x \mapsto Q_{x}$ is $\mathcal{J}_{\mathscr{C}}$-layerwise computable and $\mathscr{C}$-layerwise computable.

Proof. 1. Of course, $t_{\mathfrak{J}_{\mathscr{C}}} \stackrel{*}{<} t_{\mathscr{C}}$ as $\mathscr{C} \subseteq \mathcal{J}_{\mathscr{C}}$. Conversely, the $P \in \mathcal{J}_{\mathscr{C}}: \int t_{\mathscr{C}} \mathrm{d} P=$ $\int\left(\int t_{\mathscr{C}} \mathrm{d} Q\right) \mathrm{d} m(Q) \leq 1$ as $m$ is supported on measures in $Q \in \mathscr{C}$, and $\int t_{\mathscr{C}} \mathrm{d} Q \leq 1$ for such measures. As a result, $t_{\mathscr{C}}$ is a $\mathcal{J}_{\mathscr{C}}$-test, so $t_{\mathscr{C}} \stackrel{*}{<} t_{\mathfrak{J}_{\mathscr{C}}}$.

2. The proof is similar to the proof of Theorem 2. As $t_{\mathscr{C}}(x)=\inf _{Q \in \mathscr{C}} t_{Q}(x)$, if $x \in \mathcal{R}_{\mathscr{C}}^{n}$ then $Q_{x} \in \mathcal{R}_{m}^{n+c}$. Again, $\pi_{2}: \mathcal{R}_{m}^{n+c} \times \mathcal{R}_{\mathscr{C}}^{n} \rightarrow \mathcal{R}_{\mathscr{C}}^{n}$ is computable and bijective so its inverse is computable and maps $x$ to $\left(Q_{x}, x\right)$. Hence $\pi_{1} \circ \pi_{2}^{-1}$ is computable on $\mathcal{R}_{\mathscr{C}}^{n}$, uniformly in $n$, i.e. it is $\mathscr{C}$-layerwise computable. As $t_{\mathscr{C}} \stackrel{*}{<} t_{\mathfrak{J}_{\mathscr{C}}}$, it is also $\mathcal{J}_{\mathscr{C}}$-layerwise computable.

Observe that for generic sequences $x, t_{\mathscr{C}}(x) \stackrel{*}{=} t_{Q_{x}}(x)$. Indeed, $t_{\mathscr{C}}(x)=$ $\inf _{P \in \mathscr{C}} t_{P}(x)=t_{Q_{x}}(x)$ as $t_{P}(x)=+\infty$ for every $P \in \mathscr{C} \backslash\left\{Q_{x}\right\}$.

\subsection{A weaker answer: finitely decomposable measures}

In the two preceding results, we need the effectivity of the ergodic decomposition. In particular situations, we still get a (weaker) result without this assumption.

Proposition 3. Let $P$ be a stationary measure such that $m_{P}$ is supported on a closed set $\mathscr{C}$ of stationary ergodic measures. For every $P$-random $x, Q_{x}$ is ergodic.

To prove it we use the following lemma.

Lemma 4. Let $X, Y$ be computable metric spaces. Let $f_{n}: X \rightarrow Y$ be uniformly computable functions that converge $P$-a.e. to a function $f$. Let $A \subseteq Y$ be a closed set such that $f(x) \in A$ for $P$-a.e. $x$. For every $P$-random $x, \lim f_{n}(x) \in A$.

Proof. It is already known if $f$ is constant $P$-almost everywhere. Let $x_{0}$ be a $P$-random point such that $\lim f_{n}\left(x_{0}\right) \notin A$. Let $B(y, r)$ be a ball with computable center and radius, containing $\lim f_{n}\left(x_{0}\right)$ and disjoint from $A$. Let $g_{n}(x)=$ $\max \left(0, r-d\left(f_{n}(x), y\right)\right)$. For $P$-almost every $x$, the sequence $g_{n}(x)$ converges to 0 , but $\lim g_{n}\left(x_{0}\right)=r-d\left(\lim f_{n}\left(x_{0}\right), y\right)>0$. 
Proof (Proof of Proposition 3). For every $n$, define $Q_{n}: X \rightarrow \mathcal{P}(X)$ by $Q_{n}(x)=$ $\frac{1}{n}\left(\delta_{x}+\ldots+\delta_{T^{n-1} x}\right)$. A sequence $x$ is generic if and only if $Q_{n}(x)$ is weakly convergent, and in that case $Q_{x}$ is the limit of $Q_{n}(x)$. The functions $Q_{n}$ are uniformly computable. As $Q_{x} \in \mathscr{C}$ for $P$-almost every $x, Q_{x} \in \mathscr{C}$ for every $P$-random $x$ by Lemma 4 .

For instance, if $P$ has a finite decomposition, i.e. if $P=\sum_{i=1}^{n} \alpha_{i} P_{i}$ where $\alpha_{i} \in[0,1], \sum_{i} \alpha_{i}=1$ and all $P_{i}$ are ergodic, then regardless of the computability of $P, \alpha_{i}, P_{i}$, for every $P$-random $x, Q_{x} \in\left\{P_{1}, \ldots, P_{n}\right\}$ as the latter set is closed. In this particular case, $Q_{x}$ is always $m$-random.

We do not know whether every finitely decomposable measure is effectively decomposable. For instance, are there distinct non-computable ergodic measures $P_{1}, P_{2}$ such that $P:=\frac{1}{2}\left(P_{1}+P_{2}\right)$ is computable? Such a measure $P$ would be a finitely, non-effectively decomposable measure.

If a finitely, but non-effectively, decomposable measure $P$ exists, and if $x$ is $P$-random, we do not know whether $x$ is $Q_{x}$-random and we do not know whether $Q_{x}$ is $m_{P}$-random. We only know that $Q_{x}$ is ergodic.

\section{References}

1. Bienvenu, L., Day, A., Mezhirov, I., Shen, A.: Ergodic-type characterizations of algorithmic randomness. In: Computability in Europe (CIE 2010). Lecture Notes in Computer Science, vol. 6158, pp. 49-58. Springer (2010) 1, 7

2. Freer, C.E., Roy, D.M.: Computable exchangeable sequences have computable de finetti measures. In: CiE. Lecture Notes in Computer Science, vol. 5635, pp. 218231. Springer (2009) 8

3. Gács, P.: Uniform test of algorithmic randomness over a general space. Theoretical Computer Science 341, 91-137 (2005) 3

4. Gács, P.: Lecture notes on descriptional complexity and randomness. Tech. rep., Boston University (2008) 3, 9

5. Hertling, P., Weihrauch, K.: Random elements in effective topological spaces with measure. Information and Computation 181(1), 32-56 (2003) 3

6. Hoyrup, M., Rojas, C.: Applications of effective probability theory to Martin-Löf randomness. In: ICALP 2009. Lecture Notes in Computer Science, vol. 5555, pp. 549-561. Springer (2009) 1, 3, 7

7. Hoyrup, M., Rojas, C.: Computability of probability measures and Martin-Löf randomness over metric spaces. Inf. Comput. 207(7), 830-847 (2009) 3

8. Levin, L.A.: On the notion of a random sequence. Soviet Mathematics Doklady 14, 1413-1416 (1973) 3

9. Martin-Löf, P.: The definition of random sequences. Information and Control 9(6), 602-619 (1966) 1, 3

10. Nandakumar, S.: An effective ergodic theorem and some applications. In: STOC '08: Proceedings of the 40th annual ACM symposium on Theory of computing. pp. 39-44. ACM, New York, NY, USA (2008) 1

11. Phelps, R. R.: Lectures on Choquet's Theorem. Springer, Berlin (2001) 2nd edition

12. V'yugin, V.V.: Ergodic theorems for individual random sequences. Theoretical Computer Science 207(4), 343-361 (1998) 5

13. Weihrauch, K.: Computable Analysis. Springer, Berlin (2000) 1, 5, 7 